\title{
Bond Strength of Mortar mixed Activated Hwangtoh
}

\author{
Go, Seong-Seok ${ }^{*} \quad$ Yeo, Sang-Ku Lee, Hyun-Chul \\ School of Architecture, College of Engineering, Chonnam National University, Yongbong Dong $300 \mathrm{Buk}-\mathrm{Gu}$, \\ Gwangju, 500-757, Korea
}

\begin{abstract}
This study aimed to mix and test mortar incorporating activated Hwangtoh to improve the Hwangtoh brick bond strength of brick structures. To do this, the bond strength correlation of mortar was analyzed by means of materials and experiment factors and levels, and the optimum conditions were suggested after analyzing the physical properties of brick and the mix ratio of mortar and additive. Furthermore, the compressive strength and bond strength were found to be in inverse proportion, and in terms of the materials and mixing level, W/C ratio, substitution ratio of activated Hwangtoh, and fine aggregate grading were shown to have a considerable influence on the strength. In conclusion, the optimum mixing conditions to improve the bond strength are found to set $\mathrm{W} / \mathrm{C}$ ratio at $65 \%$ and replacmenet ratio of activated Hwangtoh at $10 \%$.
\end{abstract}

Keywords : activated hwangtoh, mortar, hwangtoh brick, brick bond strength, compressive strength

\section{Introduction}

\subsection{Research background and objective}

To address issues such as the natural resources crisis, the destruction of the natural environment and the ecosystem, the depletion of natural resources, and the emission of pollutants from construction materials, environmental preservation and green building construction have been given much attention recently[1,2]. In addition, the sustainable construction movement has been accelerated, with environment-related government agencies and organizations, construction-related associations, construction companies and construction material manufacturers working together in advanced countries and making efforts to limit the use of

Received : October 24, 2011

Revision received : August 22, 2012

Accepted : August 29, 2012

* Corresponding author : Go, Seong-Seok

[Tel: +82-62-530-1643, E-mail: ssgo@jnu.ac.kr]

(c)2012 The Korea Institute of Building Construction, All rights reserved. pollutant-emitting construction materials, utilize natural resources, develop non-polluting construction materials, and recycle construction waste[3]. In addition, a variety of environmental and functional construction materials are currently being used to enhance the eco-friendly features, including structures mainly built with Hwangtoh bricks, boards and finishing materials, to name a few.

Unlike general sintered bricks, Hwangtoh bricks are manufactured through a process of natural drying after pressing, and are known to have good eco-friendly features, including a high absorption rate, humidity control, and deodorization performance. For this reason, Hwangtoh bricks have become popular in building construction as material for internal and external walls as the demand for suburban houses rises. Since Hwangtoh bricks are mostly used in a wet condition, the volume of adhesives used for masonry construction, including mortar and dry mortar, is also increasing accordingly. To expand the use of Hwangtoh in construction, studies are being conducted on the 
application of sintered Hwangtoh for mortar; however, there are restrictions in using ordinary cement mortar as an adhesive for Hwangtoh-brick masonry, and the strength problem of dry mortar made of ordinary Hwangtoh has been raised as an issue. Therefore, an adhesive material that is more appropriate for Hwangtoh-brick masonry should be developed, and bond strength should also be improved. In addition, if the pozzolanic property and reaction of activated Hwangtohis used to replace mortar, a non-structural element for cement brick masonry and plastering, it is expected that bond strength of Hwangtoh brick masonry will be improved, and the environmental load arising from cement production reduced.

With these facts in mind, the mortar was mixed using activated Hwangtoh to improve bond strength of Hwangtoh brick masonry and reduce environmental load of cement use. The properties of mortar were analyzed by mix proportion, and a bond strength test was performed to analyze the correlation between application condition and strength, and ultimately provide appropriate guidance for the use of mortar in a Hwangtoh brick structure.

\subsection{Research method and scope}

Literature was reviewed to understand the properties of Hwangtoh, and appropriate test factors and mix proportion were set for activated Hwangtoh mortar mix after a preliminary test. Absorption rate, replacement ratio of activated Hwangtoh, aggregate, grading of fine aggregate, polymers, etc. were determined as factors influencing the properties of activated Hwangtoh mortar. The research scope was limited in order to analyze basic properties of the activated Hwangtoh mortar such as flow and compressive strength by mix proportion, the bond strength at application to Hwangtoh bricks, and the correlation between the bond strength and each influencing factor.

\section{Theoretical review}

\subsection{Components and formation of Hwangtoh}

Hwangtoh, an indigenous natural loess, is mostly composed of minerals with the exception of the water and air in the pores. There might be a small amount of amorphous substance in thesolid that has not yet turned into mineral, such as plant roots, and microorganisms like bacteria, but the proportion of such elements is very small, and the main components of Hwangtoh can be considered as minerals, mostly consisting of particles weathered from granite, diorite, quartz porphyry, felsophyre, and alunite before and after the end of the Cretaceous period. Hwangtoh can be found all over Korea but is especially plentifulin kaolin production districts. A large amount of high quality Hwangtoh is produced from the surface layer of kaolin in Hadong, Goseong and Sancheong, Gyeongsangnam-do, Korea. The Hwangtoh produced in the districts is made in the course of decomposition of quartz trachyte, andesite and granite by weathering and hydrothermal process.

The particles of Hwangtoh include a variety of minerals such as quartz, feldspar, mica, and calcite. A light-yellow sediment, it can be divided into yellow and red soil. It consists of the following minerals, in the following ratios: $50-60 \%$ of silica $\left(\mathrm{SiO}_{2}\right)$ with large pore size, $8-12 \%$ of alumina $\left(\mathrm{Al}_{2} \mathrm{O}_{3}\right), 5-12 \%$ of lime (CaO), 2-6\% oxide of magnesium ( $\mathrm{MgO}), 2-4 \%$ ferric oxide $\left(\mathrm{Fe}_{2} \mathrm{O}_{3}\right), 0.8-1.1 \%$ ferric oxide $\left(\mathrm{Fe}_{2} \mathrm{O}_{3}\right)$, and $0.5 \%$ each of titanium dioxide $\left(\mathrm{TiO}_{2}\right)$ and manganese oxide $(\mathrm{MnO})$. The chemical components and formation of Hwangtoh have much in common with those of kaolin, and contain relatively more iron oxide. Iron oxide is one of the transition metal oxides, andis reported to have 95 percent far-IR emissivity. The properties of 
Hwangtoh may vary slightly depending on factors in the district in which the Hwangtoh was formed, such as the geologic environment, hydrologic environment, geological features and climate. Hwangtoh is classified as halloysite, one of the kaolin sub group of minerals, and it contains $\mathrm{SiO}_{2}, \mathrm{Al}_{2} \mathrm{O}_{3}$, and $\mathrm{Fe}_{2} \mathrm{O}_{3}$ as main components, which are similar to concrete admixture, and also has a pozzolanic property. It is also known as an eco-friendly material with advantages such as thermal storage, self-cleaning power, deodorization, anti-bacterial function, and far-IR emissivity, and is generally known to be good for the human body[4,5]. In addition, when sintering at a high temperature, $\mathrm{SiO}_{2}$ and $\mathrm{Al}_{2} \mathrm{O}_{3}$ of the activated organics have a pozzolanic reaction with $\mathrm{Ca}(\mathrm{OH})_{2}$, generating $\mathrm{CSH}$ gel and CASH gel. For this reason, it is also highly evaluated as a possible concrete admixture[6].

\subsection{Bond strength of mortar}

The general properties of mortar may vary depending on mix proportion, initial flow and use of admixture. The masonry unit is a large rectangular wall made through acombination of bricks and mortar. It has been found in many studies $[7,8]$ that the stronger the combination between bricks and mortar, the higher the strength of the brick wall. Hence, it is noted that the bond strength of mortar in the masonry unit has a great impact on permeability and structural preservation. If the bond strength improves with the strength of masonry wall kept at a required level, it can contribute to the safety and durability ofthe concrete wall. For this reason, advanced countries have already established the criteria for bond strength test of mortar[9], and many studies have been conducted in compliance with the criteria. Based on the W/C theory that it is best to mix mortar with the minimum amount of water that satisfies the workability requirements, mortar is made for masonry work. Considering the bond strength and compressive strength ofmortar, bond strength should be considered more than anything else in the properties of mortar used in masonry work. In terms of flow, compressive strength of mortar should first be secured, and then the compressive strength should be taken into account[10]. That is, the water in the mortar is absorbed by the upper and lower masonry bricks, and it is important to secure flowability of mortar for integrity of the masonry wall. Thisis why the bond strength is considered important in the masonry unit.

\section{Test plan and method}

\subsection{Test materials and properties}

The cement used in this test is ordinary Portland cement with relative density of $3.15 \mathrm{~kg} / \mathrm{m}^{3}$ and Blaine fineness of $3,466 \mathrm{~cm}^{2} / \mathrm{g}$. The fine aggregate is river sand with fineness modulus (FM) of $2.56 \mathrm{~mm}$ and up to $5 \mathrm{~mm}$. The aggregate was classified into 4 types based on the standard grading scale through the test for sieve analysis (see Figure 1). The Hwangtoh used is natural Hwangtoh powder with an average particle size of $13 \mu \mathrm{m}$. Hwangtoh was activated after sintering it for 1 hour at $800^{\circ} \mathrm{C}$, and then it was cooled rapidly. Fly ash (FA) and blast furnace slag (BFS) were used as admixture, and SBR latex and epoxy resin were used as polymer. The absorption rate of Hwangtoh bricks used in the test was $15 \%$ at room temperature. When dampened, the absorption rate of the bricks improved to be $18 \%$ at 15 minutes and $20 \%$ at 30 minutes. Table 1 indicates the property of materials used in the test. Tables 2 through 6 indicate the chemical composition of Hwangtoh, and the physical properties of cement, aggregate, and LBR latex and epoxy resin, respectively. 
Table 1. Properties of materials

\begin{tabular}{|c|c|c|c|c|c|c|}
\hline Material & $\begin{array}{l}\text { hwangtoh } \\
\text { brick }\end{array}$ & Cement & Fine aggregate & $\begin{array}{l}\text { Activated } \\
\text { Hwangtoh }\end{array}$ & Admixture & Polymer(density) \\
\hline Property & $\begin{array}{c}\text { compressive } \\
\text { strength } \\
\text { 16.279MPa } \\
\text { absorption } \\
5.7 \% \\
\end{array}$ & $\begin{array}{l}\text { specific gravity }: 3.15 \\
\text { fineness:3,466cm } 2 / \mathrm{g}\end{array}$ & $\begin{array}{l}\text { river sand } \\
\text { FM: } 2.56 \\
\text { large size: } 5 \mathrm{~mm}\end{array}$ & $\begin{array}{c}\text { average size: } 13 \mu \mathrm{m} \\
\text { activation-temperature: } 800^{\circ} \mathrm{C} \\
\text { activation-time: } 1 \text { hour }\end{array}$ & $\begin{array}{c}\text { FA: } \\
\text { density: } 2.13 \\
\text { fineness:2,926cm } 2 / \mathrm{g}\end{array}$ & $\begin{array}{c}\text { SBR Latex(1.01) } \\
\text { Epoxy(1.12) }\end{array}$ \\
\hline Property & $\begin{array}{c}\text { compressive } \\
\text { strength } \\
\text { 16.279MPa } \\
\text { absorption } \\
5.7 \%\end{array}$ & $\begin{array}{l}\text { specific gravity }: 3.15 \\
\text { fineness:3,466cm } 2 / \mathrm{g}\end{array}$ & $\begin{array}{l}\text { river sand } \\
\text { FM: } 2.56 \\
\text { large size: } 5 \mathrm{~mm}\end{array}$ & $\begin{array}{c}\text { average size: } 13 \mu \mathrm{m} \\
\text { activation-temperature: } 800^{\circ} \mathrm{C} \\
\text { activation-time: } 1 \text { hour }\end{array}$ & $\begin{array}{c}\text { BFS: } \\
\text { density: } 2.93 \\
\text { fineness:4,204cm2/g }\end{array}$ & $\begin{array}{c}\text { SBR Latex(1.01) } \\
\text { Epoxy(1.12) }\end{array}$ \\
\hline
\end{tabular}

Table 2. Chemical composition of Hwangtoh

\begin{tabular}{cccc}
\hline $\begin{array}{c}\text { chemical } \\
\text { composition }\end{array}$ & ratio(\%) & $\begin{array}{c}\text { chemical } \\
\text { composition }\end{array}$ & ratio(\%) \\
\hline $\mathrm{SiO}_{2}$ & 42.5 & $\mathrm{CaO}$ & 0.57 \\
$\mathrm{Al}_{2} \mathrm{O}_{3}$ & 36.6 & $\mathrm{~K}_{2} \mathrm{O}$ & 0.41 \\
$\mathrm{MgO}$ & 0.69 & $\mathrm{Na}_{2} \mathrm{O}$ & 0.18 \\
\hline
\end{tabular}

Table 3. Physical properties of cement

\begin{tabular}{|c|c|c|c|c|c|c|c|}
\hline \multirow{2}{*}{ density } & \multirow{2}{*}{$\begin{array}{l}\text { finen } \\
\text { ess } \\
(\mathrm{cm} / \mathrm{g})\end{array}$} & \multirow{2}{*}{$\begin{array}{l}\text { stability } \\
(\%)\end{array}$} & \multicolumn{2}{|c|}{$\begin{array}{c}\text { setting } \\
\text { time[gilmore(min)] }\end{array}$} & \multicolumn{3}{|c|}{ compressive strength(Mpa) } \\
\hline & & & first & end & 3cay & 7day & 28day \\
\hline 3.15 & 3,466 & 0.10 & 303 & 460 & 21.9 & 26.8 & 35.2 \\
\hline
\end{tabular}

Table 4. Physical properties of fine-aggregate

\begin{tabular}{|c|c|c|c|c|c|}
\hline \multirow[b]{2}{*}{ Type } & \multirow[b]{2}{*}{ (F.M) } & \multicolumn{2}{|c|}{ density $\left(\mathrm{g} / \mathrm{cm}^{\mathrm{m}}\right)$} & \multirow[b]{2}{*}{$\begin{array}{c}\text { absorptio } \\
\text { n ratio } \\
(\%)\end{array}$} & \multirow[b]{2}{*}{$\begin{array}{c}\text { size } \\
\text { classification } \\
\text { level }\end{array}$} \\
\hline & & $\begin{array}{c}\text { overdry } \\
\text { condition }\end{array}$ & $\begin{array}{l}\text { surface } \\
\text { drying } \\
\text { condition }\end{array}$ & & \\
\hline $\begin{array}{l}\text { River } \\
\text { sand }\end{array}$ & 2.56 & 2.51 & 2.55 & 1.86 & 4 \\
\hline
\end{tabular}

Table 5. Physical properties of SBR Latex

\begin{tabular}{cccccc}
\hline Type & $\begin{array}{c}\text { Solid } \\
(\%)\end{array}$ & $\begin{array}{c}\text { Viscosity } \\
\left(25^{\circ} \mathrm{C},\right. \\
\mathrm{CP})\end{array}$ & $\begin{array}{c}\text { Surface } \\
\text { Tension } \\
(\text { dyne } / \mathrm{cm})\end{array}$ & $\begin{array}{c}\mathrm{pH} \\
\text { value }\end{array}$ & $\begin{array}{c}\text { Density } \\
\left(\mathrm{g} / \mathrm{cm}^{2}\right)\end{array}$ \\
\hline Liquid & $\begin{array}{c}50 \pm \\
2\end{array}$ & 70 & 35 & 10.3 & 1.01 \\
\hline
\end{tabular}

Table 6. Physical properties of Epoxy resin

\begin{tabular}{ccccc}
\hline $\begin{array}{c}\text { Epoxy } \\
\text { Equivalent }\end{array}$ & $\begin{array}{c}\text { Molecular } \\
\text { Weight }\end{array}$ & $\begin{array}{c}\text { Hue } \\
(\text { Gardner })\end{array}$ & $\begin{array}{c}\text { Density } \\
\left(\mathrm{g} / \mathrm{cm}^{3}\right)\end{array}$ & $\begin{array}{c}\text { Viscosity } \\
\left(\mathrm{mPas}, 20^{\circ} \mathrm{C}\right)\end{array}$ \\
\hline 170 & 340 & $<1$ & 1.19 & 3500 \\
\hline
\end{tabular}

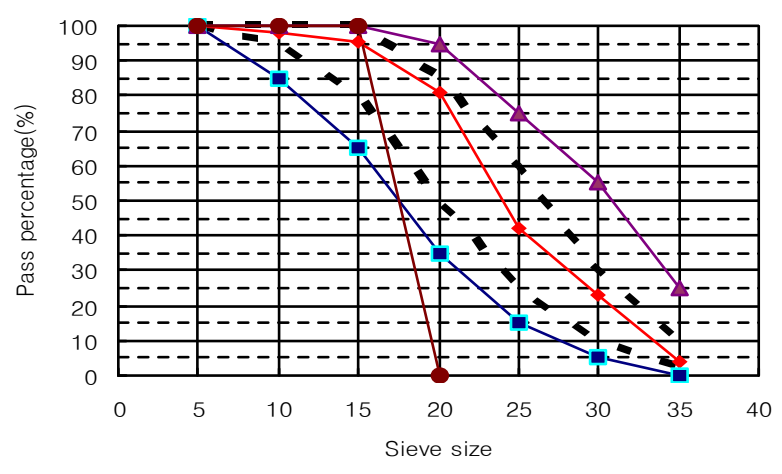

Figure 1. Fine aggregate grading classification

\subsection{Test plan and method}

To conduct the test, the absorption rate of Hwangtoh brick and the property influencing factors of the activated Hwangtoh mortar were first set as parameters. The OPC mortar and existing dry Hwangtoh mortar were also tested as controls. In terms of absorption rate, dampening time was set to be 15 and 30 minutes, and the water to cement ratio (W/C) was set to $60 \%$ based on a fundamental experiment. For the sake of comparison, two different levels of W/C were also set, at 55\% and 65\%. In addition, natural Hwangtoh and activated Hwangtoh were used as substitute for cement to compare the properties of activated Hwangtoh mortar with those of the others. The activated Hwangtoh sintered for one hour at $800^{\circ} \mathrm{C}$ was used, as it showed the best performance in previous studies. The replacement ratio of activated 
Hwangtoh was set at five different levels: 5\%, 10\%, $15 \%$, 20\%, and 25\%, to analyze appropriate replacement condition, reduce the volume of cement used and improve the performance as a result. FA and BFS were used as admixture. The two polymers of epoxy resin and SBR latex were selected and used with admixture. The overall experiment factors and level are indicated in Table 7.

Table 7. Experiment factors and level

\begin{tabular}{ccc}
\hline Experimental factor & $\begin{array}{c}\text { Le } \\
\text { vel }\end{array}$ & Type \\
\hline W/C & 3 & $55 \%, 60 \%, 65 \%$ \\
Hwangtoh & 2 & Normal(H), Activated(AH) \\
standard curve(in:1, out \\
:2), singular(1) \\
Aggregate size & 4 & 15 min, 30min \\
Brick absorption & 2 & $5 \%, 10 \%, 15 \%, 20 \%, 25 \%$ \\
Hwangtoh & 5 & Fly ash, Blast furnace slag \\
substitution & 2 & Flow15cm, 18cm \\
Admixture & 2 & Epoxy, SBR-latex \\
Hwangtoh Ready & 1 & Mixed Dry mortar \\
Polymer & 2 &
\end{tabular}

The mix and test specimen are shown in Table 8. The specimen was manufactured at a different proportion of experiment factors by group. A total of 26 specimens were made in 9 groups.

The mortar was mixed using a machine mixing method, using different proportions of experiment factors according to the experiment schedule. The flowability of the mortar was measured first, and then specimens were made for compressive strength and bond strength tests. The specimen for compressive strength test was molded in a cubic form of $5 \mathrm{~cm} \times 5 \mathrm{~cm} \times 5 \mathrm{~cm}$, and cured in a wet condition. The specimen for bond strength test of the masonry unit was manufactured, as shown in Figure 2, by placing the upper and lower bricks crossed in the middle using a plate. More specifically, the lower bricks were placed first, and the plastic glue mold for bricks was separately manufactured to a size of $9 \mathrm{~cm} \times 9 \mathrm{~cm} \times 1 \mathrm{~cm}$, on which the upper bricks were crossed with the lower bricks.
The specimens were cured for 28 days at room temperature, and tested. The specimen were placed to be crossed in a test machine and tested by putting the load until the masonry unit fell to analyze the characteristics of bond strength of the mortar as calculated using Equation (1).

Bond strength $=$ load/bonded area $(\mathrm{MPa})-$ Equation (1)

Table 8. Mix and test specimen

\begin{tabular}{|c|c|c|c|c|c|c|c|c|c|}
\hline \multirow{5}{*}{$\begin{array}{l}\text { Series } \\
\text { I. Plain } \\
\text { mortar } \\
(\text { W/C })\end{array}$} & \multirow{2}{*}{\multicolumn{2}{|c|}{$\begin{array}{c}\text { No. } \\
\text { Specimen }\end{array}$}} & \multirow{3}{*}{$\begin{array}{c}\text { W/C } \\
(\%) \\
55\end{array}$} & \multicolumn{4}{|c|}{ Weight(kg/m3) } & \multicolumn{2}{|c|}{ Admi Polym } \\
\hline & & & & \multicolumn{2}{|c|}{ Cement Water } & \multirow{2}{*}{$\begin{array}{c}\text { Sand } \\
566\end{array}$} & \multirow{2}{*}{$\begin{array}{c}\text { Hwa } \\
\text { ngto } \\
\text { h }\end{array}$} & \multirow{2}{*}{$\begin{array}{c}\text { xture } \\
(\mathrm{kg}) \\
-\end{array}$} & \multirow{2}{*}{$\begin{array}{c}\text { er } \\
(\mathrm{kg})\end{array}$} \\
\hline & 1 & S-55 & & 159 & 275 & & & & \\
\hline & 2 & $S-60$ & 60 & 155 & 293 & 552 & 0 & - & - \\
\hline & 3 & $S-65$ & 65 & 151 & 310 & 539 & 0 & - & - \\
\hline \multirow{3}{*}{$\begin{array}{l}\text { II. Natural } \\
\text { Hwangtoh } \\
\text { Mortar }\end{array}$} & 4 & $\mathrm{H}-55$ & 55 & 118 & 273 & 562 & 47 & - & - \\
\hline & 5 & $\mathrm{H}-60$ & 60 & 118 & 273 & 562 & 47 & - & - \\
\hline & 6 & $\mathrm{H}-65$ & 65 & 113 & 307 & 535 & 45 & - & - \\
\hline \multirow{3}{*}{$\begin{array}{c}\text { III. } \\
\text { Activated } \\
\text { Hwangtoh } \\
\text { mortar }\end{array}$} & 7 & $\mathrm{AH}-55$ & 55 & 118 & 273 & 562 & 47 & - & - \\
\hline & 8 & $\mathrm{AH}-60$ & 60 & 115 & 291 & 548 & 46 & - & - \\
\hline & 9 & $\mathrm{AH}-65$ & 65 & 113 & 307 & 535 & 45 & - & - \\
\hline \multirow{5}{*}{$\begin{array}{l}\text { IV. } \\
\text { Substitutio } \\
\mathrm{n} \text { ratio of } \\
\text { activated } \\
\text { Hwangtoh }\end{array}$} & 10 & $\mathrm{AH}-5$ & 60 & 147 & 292 & 552 & 9 & - & - \\
\hline & & $\mathrm{AH}-10$ & 60 & 139 & 292 & 551 & 18 & - & - \\
\hline & 12 & $\mathrm{AH}-15$ & 60 & 131 & 291 & 550 & 27 & - & - \\
\hline & 13 & $\mathrm{AH}-20$ & 60 & 123 & 291 & 549 & 37 & - & - \\
\hline & 14 & $\mathrm{AH}-30$ & 60 & 107 & 290 & 548 & 55 & - & - \\
\hline \multirow{4}{*}{$\begin{array}{c}\mathrm{V} . \\
\text { Aggregate }\end{array}$} & 15 & $\ln 1$ & 60 & 115 & 291 & 548 & 46 & - & - \\
\hline & & out 1 & 60 & 115 & 291 & 548 & 46 & - & - \\
\hline & & out 2 & 60 & 115 & 291 & 548 & 46 & - & - \\
\hline & 18 & $\begin{array}{c}\text { singular } \\
1\end{array}$ & 60 & 115 & 291 & 548 & 46 & - & - \\
\hline \multirow{2}{*}{$\begin{array}{l}\text { VI.Brick } \\
\text { absorption }\end{array}$} & 19 & $15 \mathrm{~min}$ & 60 & 115 & 291 & 548 & 46 & - & - \\
\hline & 20 & $30 \mathrm{~min}$ & 60 & 115 & 291 & 548 & 46 & - & - \\
\hline \multirow{2}{*}{$\begin{array}{l}\text { VII.Ready } \\
\text { mixed dry } \\
\text { mortar }\end{array}$} & & flow15 & - & 0 & 946 & 54 & 0 & - & - \\
\hline & 22 & flow18 & - & 0 & 936 & 64 & 0 & - & - \\
\hline \multirow{2}{*}{$\begin{array}{l}\text { VIII. Mortar } \\
\text { mixing } \\
\text { admixture }\end{array}$} & & $F A-10$ & 60 & 115 & 291 & 548 & 32 & 14 & - \\
\hline & 24 & BFS-10 & 60 & 115 & 291 & 548 & 32 & 14 & - \\
\hline \multirow{2}{*}{$\begin{array}{l}\text { IX. Mortar } \\
\text { mixing } \\
\text { polymer }\end{array}$} & 25 & EPO-5 & 60 & 117 & 276 & 555 & 46 & - & 7 \\
\hline & 26 & SBR-5 & 60 & 117 & 276 & 555 & 46 & - & 7 \\
\hline \multicolumn{10}{|c|}{$\begin{array}{l}\text { ※ Material condition } \\
\text { - Cement(Ordinary por } \\
\text { - Cement brick(190×90 } \\
\text { - Natural Hwangtoh(ter } \\
\text { - Activated Hwangtoh(t } \\
\text { cooling: fast) } \\
\text { - superplasticizer } 2 \%\end{array}$} \\
\hline
\end{tabular}


SD: standard deviation, V: variation

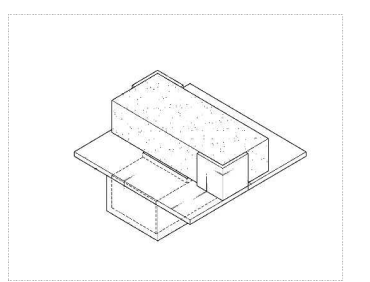

(a) Design of mortar mold(1)

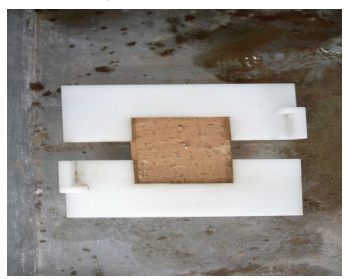

(c) Mortar $\operatorname{pad}(1)$

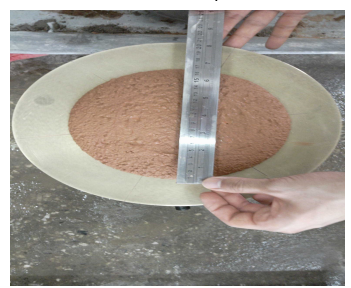

(e) Flow test

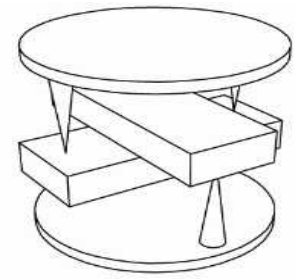

(g) Bond strength test(1)

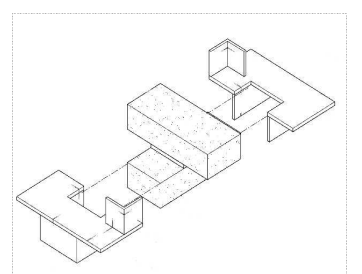

(b) Design of mortar mold(2)

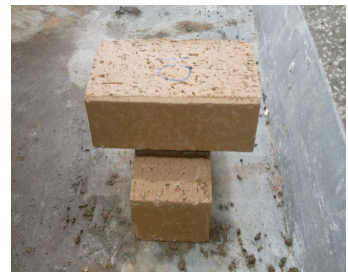

(d) Mortar pad(2)

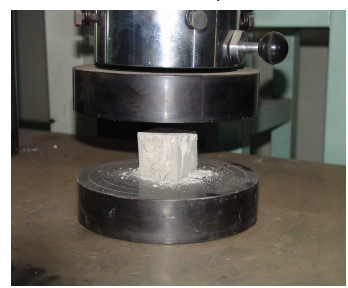

(f) Compression strength test

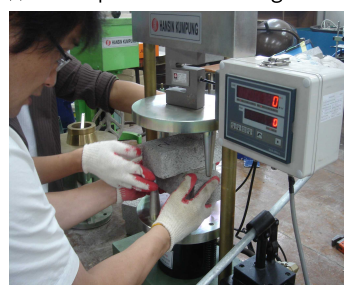

(h) Bond strength test(2)

Figure 2. Method of testing

\section{Test results and considerations}

A comparative analysis was performed to evaluate compressive strength and bond strength according to property influencing factors to present appropriate conditions of activated Hwangtoh mortar for strength improvement and utilization in Hwangtoh masonry brick.

\subsection{Test results}

Activated Hwangtoh mortar was tested at different levels of factors influencing the bond strength property. The compressive and bond strength test

results are indicated in Tables 9 and 10, respectively. Compressive strength, brick bond strength and strength correlation coefficient were measured at 28 days, and the test results were drawn based on the data. It was found that as the W/C increased, the compressive strength decreased by $25 \%$ from $24.47 \mathrm{MPa}$ to $18.21 \mathrm{MPa}$, or in the ratio of $1.021 \sim 0.760$ compared to at $60 \% \mathrm{~W} / \mathrm{C}$, while the bond strength increased by $35 \%$ from $0.5 \mathrm{MPa}$ to $0.69 \mathrm{MPa}$, or in the ratio of $0.8024 \sim 1.149$ compared to at $60 \% \mathrm{~W} / \mathrm{C}$.

Table 9. Result of compressive strength test

\begin{tabular}{|c|c|c|c|c|c|c|c|}
\hline & \multirow[b]{2}{*}{$\begin{array}{c}\text { W/C } \\
(\%)\end{array}$} & \multicolumn{6}{|c|}{ Compressive strength(28days) } \\
\hline & & $\begin{array}{l}\text { Strength } \\
(\mathrm{MPa})\end{array}$ & $\mathrm{SD}$ & V & Coefficient & $\begin{array}{c}\mathrm{H} / \mathrm{S} \\
\mathrm{AH} / \mathrm{S}\end{array}$ & $\mathrm{AH} / \mathrm{H}$ \\
\hline S55 & 55 & 24.47 & 1.02 & 1.05 & 1.021 & & \\
\hline S60 & 60 & 23.96 & 0.36 & 0.13 & 1.000 & & \\
\hline S65 & 65 & 18.21 & 0.95 & 0.91 & 0.760 & & \\
\hline H55 & 55 & 20.68 & 0.08 & 0.01 & 1.104 & 0.845 & \\
\hline $\mathrm{H} 60$ & 60 & 18.74 & 0.24 & 0.06 & 1.000 & 0.782 & \\
\hline H65 & 65 & 16.85 & 0.29 & 0.08 & 0.899 & 0.925 & \\
\hline $\mathrm{AH} 55$ & 55 & 29.00 & 0.23 & 0.05 & 1.140 & 1.185 & 1.402 \\
\hline $\mathrm{AH} 60$ & 60 & 25.45 & 1.13 & 1.28 & 1.000 & 1.062 & 1.358 \\
\hline AH65 & 65 & 21.35 & 0.75 & 0.56 & 0.839 & 1.172 & 1.267 \\
\hline $\mathrm{AHO}$ & 60 & 23.96 & 0.36 & 0.13 & 0.981 & & \\
\hline $\mathrm{AH} 5$ & 60 & 22.86 & 0.22 & 0.05 & 0.936 & & \\
\hline $\mathrm{AH} 10$ & 60 & 23.14 & 1.10 & 1.21 & 0.947 & & \\
\hline $\mathrm{AH} 15$ & 60 & 24.43 & 0.86 & 0.74 & 1.000 & & \\
\hline $\mathrm{AH} 20$ & 60 & 26.00 & 0.83 & 0.69 & 1.064 & & \\
\hline $\mathrm{AH} 25$ & 60 & 26.09 & 0.30 & 0.09 & 1.068 & & \\
\hline $\mathrm{AH} 30$ & 60 & 25.68 & 0.21 & 0.05 & 1.051 & & \\
\hline AG I1 & 60 & 20.98 & 1.87 & 3.48 & 1.000 & & \\
\hline AG O1 & 60 & 22.34 & 0.11 & 0.01 & 1.064 & & \\
\hline AG O2 & 60 & 20.60 & 3.11 & 9.68 & 0.981 & & \\
\hline AG S1 & 60 & 19.75 & 0.77 & 0.59 & 0.941 & & \\
\hline FA10 & 60 & 23.52 & 0.13 & 0.02 & 1.000 & & \\
\hline BFS10 & 60 & 26.83 & 0.80 & 0.64 & 1.141 & & \\
\hline EPO5 & 60 & 24.79 & 0.01 & 0.00 & 1.000 & & \\
\hline SBR5 & 60 & 24.92 & 0.70 & 0.49 & 1.005 & & \\
\hline
\end{tabular}


Table 10. Result of brick bond strength test

\begin{tabular}{|c|c|c|c|c|c|c|}
\hline & \multicolumn{6}{|c|}{ Brick bond strength(28days) } \\
\hline & $\begin{array}{c}\text { Strength } \\
(\mathrm{MPa})\end{array}$ & SD & V & Coefficient & $\begin{array}{l}\mathrm{H} / \mathrm{S} \\
\mathrm{AH} / \mathrm{S}\end{array}$ & $\mathrm{AH} / \mathrm{H}$ \\
\hline S55 & 0.50 & 0.04 & 0.00 & 0.824 & & \\
\hline S60 & 0.60 & 0.01 & 0.00 & 1.000 & & \\
\hline S65 & 0.69 & 0.02 & 0.00 & 1.149 & & \\
\hline $\mathrm{H} 55$ & 0.40 & 0.00 & 0.00 & 0.910 & 0.800 & \\
\hline $\mathrm{H} 60$ & 0.44 & 0.00 & 0.00 & 1.000 & 0.725 & \\
\hline $\mathrm{H} 65$ & 0.52 & 0.00 & 0.00 & 1.192 & 0.752 & \\
\hline AH55 & 0.39 & 0.03 & 0.00 & 0.931 & 0.780 & 0.985 \\
\hline $\mathrm{AH} 60$ & 0.42 & 0.02 & 0.00 & 1.000 & 0.700 & 0.963 \\
\hline AH65 & 0.54 & 0.02 & 0.00 & 1.280 & 0.782 & 1.035 \\
\hline $\mathrm{AHO}$ & 0.60 & 0.01 & 0.00 & 0.828 & & \\
\hline $\mathrm{AH} 5$ & 0.91 & 0.04 & 0.00 & 1.246 & & \\
\hline $\mathrm{AH} 10$ & 0.74 & 0.03 & 0.00 & 1.020 & & \\
\hline $\mathrm{AH} 15$ & 0.73 & 0.11 & 0.01 & 1.000 & & \\
\hline $\mathrm{AH} 20$ & 0.60 & 0.01 & 0.00 & 0.821 & & \\
\hline $\mathrm{AH} 25$ & 0.42 & 0.02 & 0.00 & 0.579 & & \\
\hline $\mathrm{AH} 30$ & 0.48 & 0.07 & 0.00 & 0.658 & & \\
\hline$A G \mid 1$ & 0.77 & 0.00 & 0.00 & 1.000 & & \\
\hline AG O1 & 0.66 & 0.02 & 0.00 & 0.857 & & \\
\hline AG O2 & 0.62 & 0.08 & 0.00 & 0.805 & & \\
\hline AG S1 & 0.05 & 0.01 & 0.00 & 0.065 & & \\
\hline $15 \mathrm{~min}$ & 0.51 & 0.00 & 0.01 & 1.000 & & \\
\hline $30 \mathrm{~min}$ & 0.77 & 0.11 & 0.00 & 1.509 & & \\
\hline flow15 & 0.42 & 0.02 & 0.00 & 1.00 & & \\
\hline flow18 & 0.70 & 0.02 & 0.00 & 1.666 & & \\
\hline FA10 & 0.47 & 0.02 & 0.00 & 1.000 & & \\
\hline BFS10 & 0.58 & 0.04 & 0.00 & 1.235 & & \\
\hline EPO5 & 0.48 & 0.16 & 0.03 & 1.000 & & \\
\hline SBR5 & 0.30 & 0.03 & 0.00 & 0.629 & & \\
\hline
\end{tabular}

※ $($ bond strength $\mathrm{N} / \mathrm{cm} 2)=$, $\mathrm{P}$ :maximum $\operatorname{load}(\mathrm{N} /)$, $\mathrm{A}$ :area of brick (cm2)

\subsection{Test results and analysis}

In this section, a comparative analysis of compressive and bond strength was implemented by mix proportion with different levels of $\mathrm{W} / \mathrm{C}$, activated Hwangtoh, grading of fine aggregate, absorption rate, admixture and polymer.

\subsubsection{Analysis of strength with W/C}

When ordinary Hwangtoh was mixed, the overall strength was shown to be lower than that of Plain. More specifically, the compressive strength was shown to be 85 93\% that of Plain, while the bond strength was $75 \sim 80 \%$. It was found in the ordinary Hwangtoh mortar with replacement ratio of $25 \%$ that as the W/C increased, the compressive strength decreased, from 20.68MPa to $16.85 \mathrm{MPa}$, while bond strength increased from $0.40 \mathrm{MPa}$ to $0.52 \mathrm{MPa}$. In addition, when activated Hwangtoh was mixed, the compressive strength was shown to rise by $27 \sim 40 \%$ while the bond strength was shown to be similarto ordinary Hwangtoh mortar, at 96 103\% (Figures 3 and 4). That is, when activated Hwangtoh was mixed, the bond strength was maintained at a similar level, while the compressive strength was greatly improved in comparison with ordinary Hwangtoh mortar.

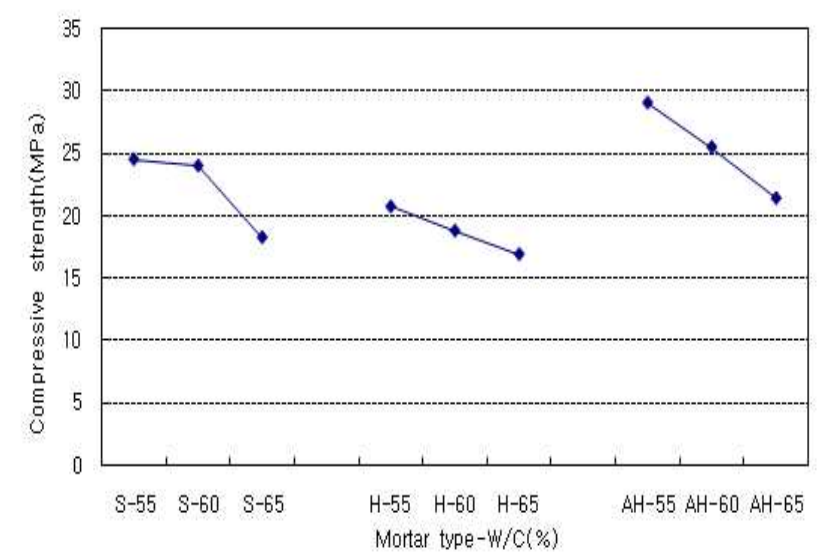

Figure 3. Analysis of the compressive strength test results

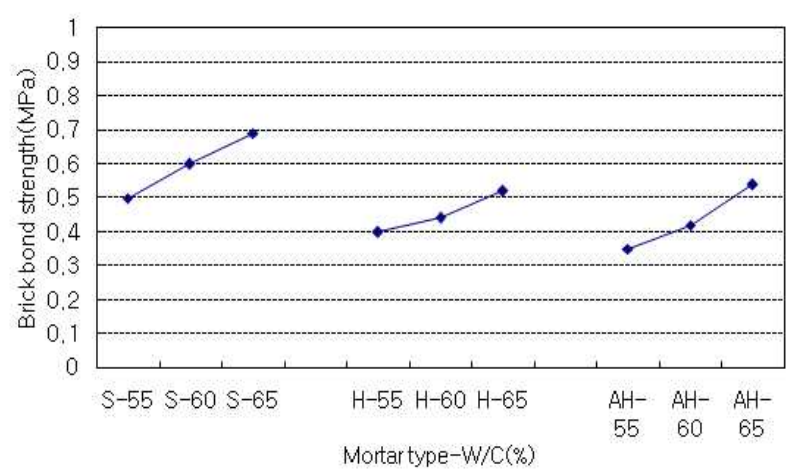

Figure 4. Analysis of the bond strength test results 


\subsubsection{Analysis of strength with replacement ratio of activated Hwangtoh}

The highest compressive strength was shown at 26.09Mpa in the activated Hwangtoh mortar with a replacement ratio of $25 \%$. As the replacement ratio increased, a gradual improvement of strength was found. On the other hand, the highest bond strength was shown at $0.91 \mathrm{MPa}$ in the activated Hwangtoh mortar with a replacement ratio of $5 \%$. As the replacement ratio increased, a gradual decrease in strength was shown. At the replacement ratio of 15\%, asharp decline in strength was shown. Taking this fact into account, an appropriate replacement ratio of Hwangtoh for masonry mortar should be set at less than 15\% (see Figures 5-6)

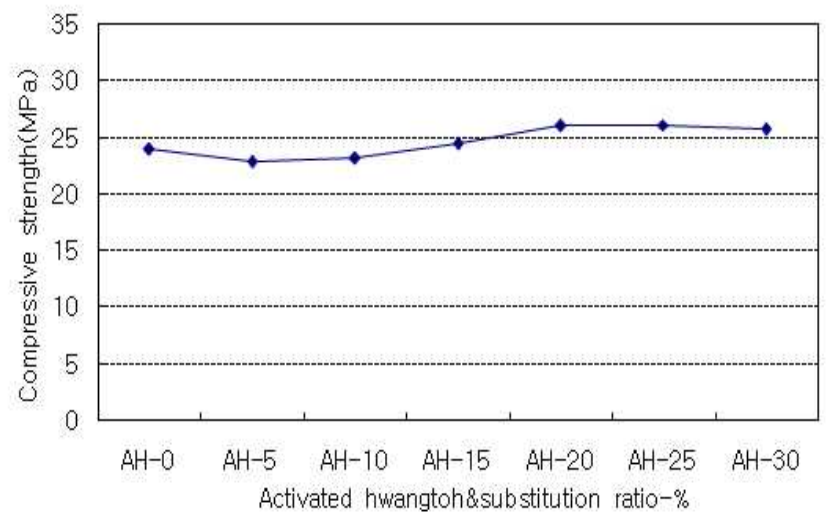

Figure 5. Analysis of the compressive strength test results

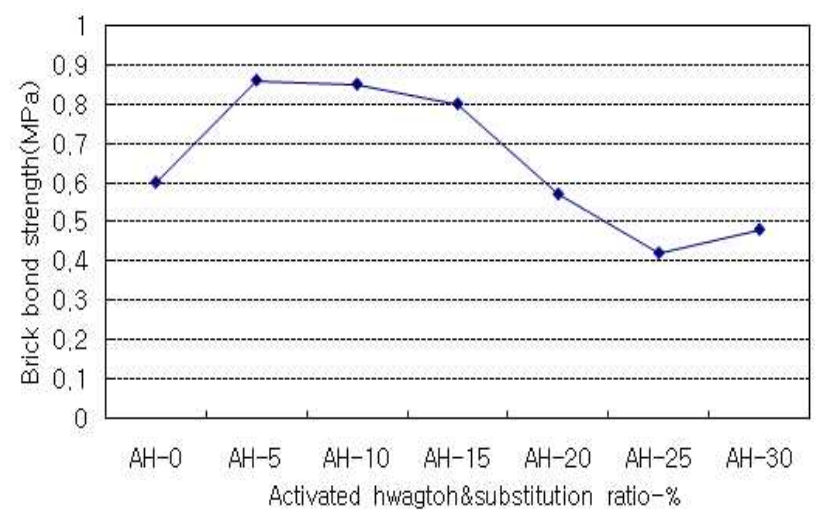

Figure 6 . Analysis of the bond strength test results

\subsubsection{Analysis of strength with grading of fine aggregate}

Thehighest compressive strength was found at
22.34MPa in AGO1 (out of the standard grading of aggregate), and was not greatly affected by grading of fine aggregate (affected by less than 7\%). However, the bond strength was greatly affected by grading. The highest bond strength was found at $0.77 \mathrm{MPa}$ in AGI1 (within the range of the standard grading of aggregate). Segregation of materials and a sharp decline in strength were observed in AGS1 (with single grain size) (Figures 7 and 8). Therefore, when mixing aggregate in mortar, the aggregate with single grain size should be avoided, and it is appropriate to use aggregate within the range of the standard grading of aggregate).



Figure 7. Analysis of the compressive strength test results

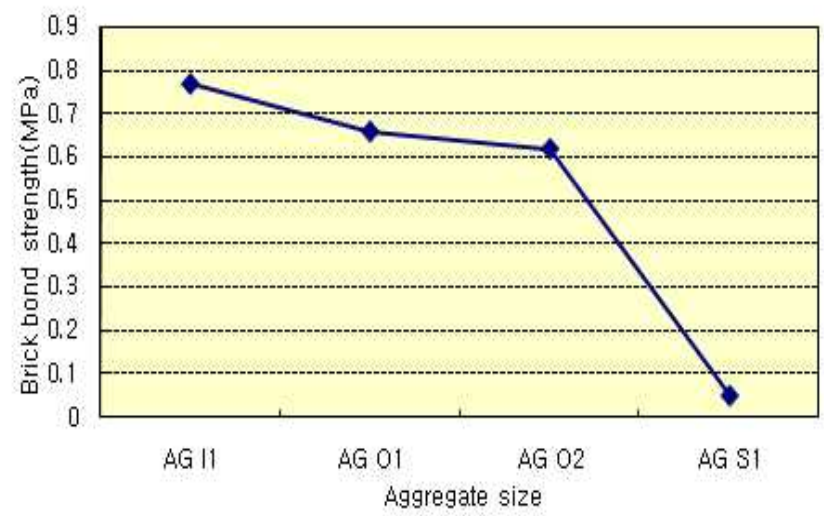

Figure 8 . Analysis of the bond strength test results

\subsubsection{Analysis of strength with absorption rate}

Through the analysis of bond strength of brickwith absorption rate of Hwangtoh brick, in the 
30-minute dampening condition, the bond strength of the brick with absorption rate of 20\% was measured at 0.77, while in the 15-minute dampening condition, the bond strength of the brick with absorption rate of $18 \%$ was measured at $0.51 \mathrm{MPa}$, which means the bond strength was improved by about $50 \%$ at $20 \%$ absorption rate in the 30-minute dampening condition compared to in the 15-minute dampening condition at 18\% absorption rate. In addition, when compared with the existing dry Hwangtoh mortar, the bond strength of the activated Hwangtoh mortar was improved by 10 20\% (see Figure 9). Therefore, to secure the integrity of the structure in brick masonry structural construction, it is believed to be advantageous to set the absorption rate as high considering the absorption rate of the brick itself. It was found that the activated Hwangtoh mortar for masonry had higher bond strength than the existing dry Hwangtoh mortar.

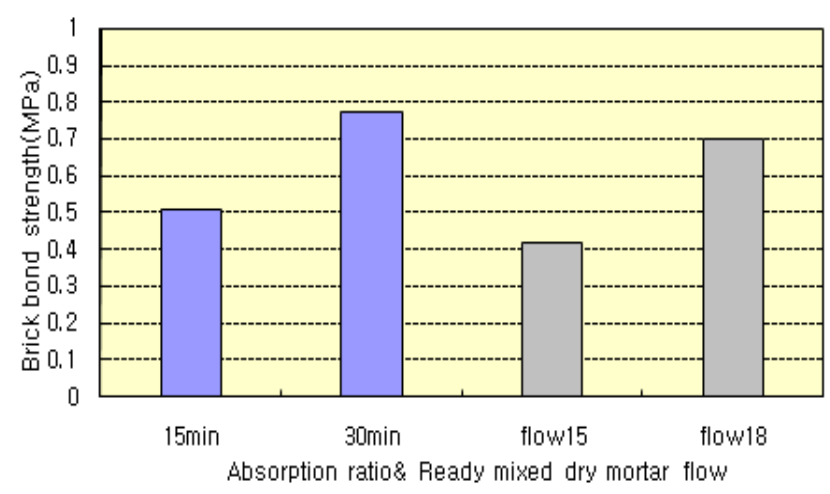

Figure 9. Analysis of the bond strength test results

\subsubsection{Analysis of strength with admixture and polymer}

The compressive strength of the mortar with BSF was 14\% higher than that of the mortar with FA. The compressive strength of the mortar with BFS was measured at 26.83MPa, compared to $23.42 \mathrm{MPa}$ for the mortar with FA. There was almost no difference in the compressive strength between the mortar with epoxy resin and the mortar with SBR latex as polymer. However, when using SBR latex, the bond strength dropped by $37 \%$ compared with when epoxy resin was used (see Figures 10 and 11). Therefore, to improve the compressive strength of activated Hwangtoh mortar it is more appropriate to use BFS than FA as admixture, and epoxy resin than SBR latex as polymer.

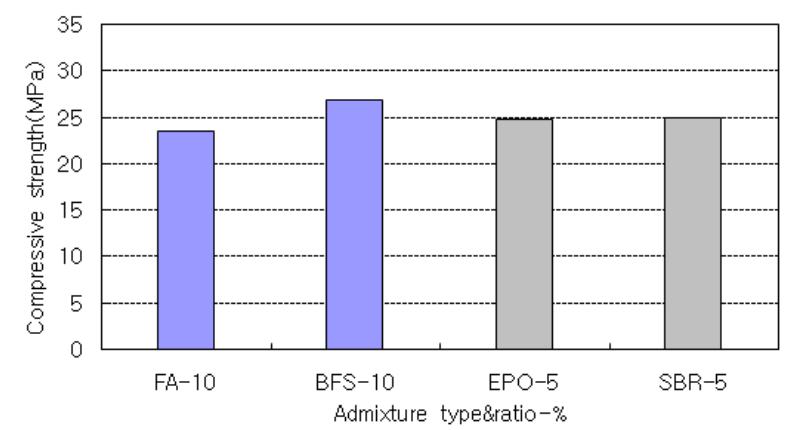

Figure 10. Analysis of the compressive strength test results

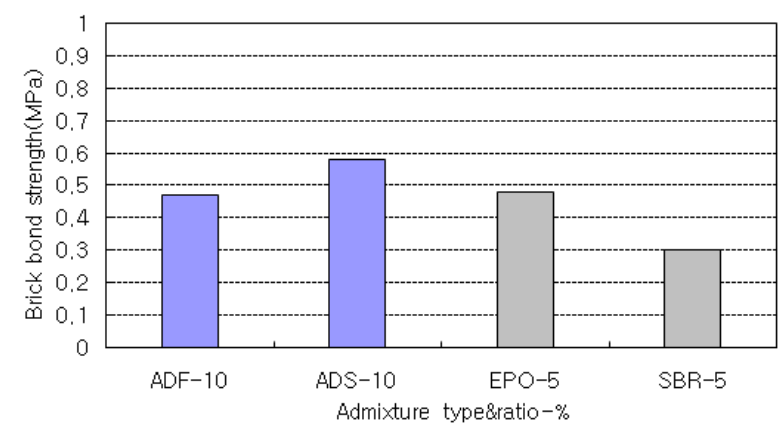

Figure 11. Brick bond strength $\mathrm{V}$

\section{Conclusion}

This study aims to present appropriate conditions of activated Hwangtoh mortar for utilization in Hwangtoh brick masonry units by implementing a comparative analysis of compressive strength and bond strength considering each parameter that might have an impact on strength properties. For the analysis, the Hwangtoh was mixed in as a replacement for cement to apply the characteristics and the pozzolanic reaction to the mortar as non-structural material for masonry and plastering. 
The research findings are as follows:

1) The ordinary Hwangtoh mortar was compared with Plain mortar. Compressive strength and bond strength of ordinary Hwangtoh mortar were shown to be 85 93\% and75 80\%, respectively, compared with those of Plain mortar. When replacing 5 10\% of cement with Hwangtoh, the bond strength greatly increased by $23 \sim 50 \%$ at a similar compressive strength level. Hwangtoh is believed to have great utility for bond strength improvement.

2) In selecting a grading of aggregate to improve the strength of mortar, if a single grading of aggregate was used, the bond strength sharply dropped by $90 \%$ compared to when using standard grading. The aggregate around the standard grading is believed to be appropriate, and improved water content through dampening is effective to improve brick bond strength.

3) When manufacturing mortar with improved compressive strength, it is most appropriate to set the mix proportion of $\mathrm{W} / \mathrm{C}$ at $60 \%$, replacement ratio of activated Hwangtoh at 20 25\%, and BFS at 10\%.

4) When manufacturing mortar with improved bond strength, it is most appropriate to set the mix proportion of $\mathrm{W} / \mathrm{C}$ at $65 \%$, and replacement ratio of activated Hwangtoh at $10 \%$.

5) There were no differences in compressive strength found when using between epoxy resin and SBR latex. When using SBR latex, the bond strength greatly decreased by $60 \%$ compared with when epoxy resin was used. To improve bond strength, it is more appropriate to use epoxy resin.

As presented in these research findings, the bond strength was shown to be low. It should also be noted that the bond strength of a brick masonry unit is greatly affected by factors other than mortar properties, such as the characteristics of the brick and the workmanship of the worker, particularly for masonry work. These should also be studied in the future to improve bond strength based on a material property analysis taking the influencing factors into account.

\section{References}

1. Ko JS, Kim BY, Park SW. An Experimental Study on the Heat Storage Properties of Phase Change Material Using Paraffin Sheets in Building. Journal of Korea Institute of Building Construction, 2011 Oct;11(5):435-41.

2. Joe WH, Lim NG. Study on Energy Performance And Economic Evaluation of Windows System with Built-in Type Blinds. Journal of Korea Institute of Building Construction. 2010 Apr;10(2):97-104.

3. Park DC, Kwon SH. Development of Eco Cementitious Building Finishing Materials Modified with Bamboo Charcoal. Journal of Korea Institute of Building Construction. 2011 Nov;11(5):452-8.

4. Go SS, Lee HC. Experimental Investigation of Mortars using Activated Hwangtoh. Journal of Construction and Building Materials. 2010 Oct;26(5):1438-45.

5. Choi HY, Hwang HZ, Kim MooH, Kim MoonH. A Study on the Development of Hwangtoh Admixture for the Application of Cement Mortar. Journal of Architectural Institute of Korea, 2000 Jun;16(6):95-102.

6. Lee HC, Go SS. A study on the Physical Properties of Mortar mixing Activated Hwangtoh. Journal of Architectural Institute of Korea. 2007 Mar;23(3):77-88.

7. John W, DE Courcy. Brick and Block masonry. London (UK): Elsevier Science Ltd; 1988. p. 162-73.

8. Arnold W. Hendry, Structural Brickwork. Hong Kong: The Macmillan Press; 1981. p. 12-5.

9. ASTM C 952. Standards Test Method for Bond Strength of Mortar. 2002. p. 18-23.

10. Yang KH, Sim J, Song JG, Lee JH, Material Properties of Slag-Based Alkali-Activated Concrete Brick-Masonry. Journal of Architectural Institute of Korea. 2011 Jan;(1):119-26. 